\title{
Über die Annahme der zeitlichen Veränderlichkeit des $\beta$-Zerfalls und die Möglichkeiten ihrer experimentellen Prüfung ${ }^{1}$
}

\author{
Von F. G. Houtermans und P. Jordan \\ Aus dem Institut für theoretische Physik der Universität Göttingen \\ (Z. Naturforschg. 1, 125-130 [1946]; eingegangen am 31. Oktober 1945)
}

\begin{abstract}
Im ersten Abschnitt wird das auf Grund der D i r a c - J or d a n schen kosmologischen Annahme zu erwartende Zerfallsgesetz für $\beta$-Zerfall und K-Einfang abgeleitet, unter Korrektur eines 1. c. ${ }^{2}$ unterlaufenen Vorzeichenfehlers. Es werden sodann Möglichkeiten der experimentellen Prüfung der Annahme einer zeitlichen Änderung des $\beta$-Zerfalls diskutiert. Im zweiten Abschnitt wird die v. We izsäckersche Annahme, daß das auf der Erde vorhandene Argonisotop 40 durch K-Einfang von $\mathrm{K}^{40}$ entstanden ist, diskutiert, und die Halbwertszeit dieses Prozesses abgeschätzt unter der Annahme, daß für den Vorrat von $A^{40}$ in der Atmosphäre die Kaliummenge in demjenigen Teil der Erdrinde verantwortlich ist, der im Laufe der Erdgeschichte den Vorgängen der Verwitterung und Sedimentbildung unterworfen war. Als Ergebnis wird unter Annahme der Halbwertszeit des $\mathrm{K}^{40}$ für $\beta$-Zerfall von $\mathrm{T}=1,42.10^{9} \mathrm{a}$ das Verhältnis $\mathrm{k}$ von K-Einfang zum Gesamtzerfall des $\mathrm{K}^{40}$ als Funktion des Alters der Atmosphäre gegeben. Für ein Alter der Atmosphäre von 3,5.109 a ergibt sich $\mathrm{k}=0,56$ nach dem klassischen Zerfallsgesetz, nach (2), wenn das Weltalter als praktisch ebenso groß angesehen wird, $\mathrm{k}=0,26$. Ferner wird festgestellt, daß unter der erwähnten geologischen Annahme der von T h o m p s o n und R o w land s gegebene experimentelle Wert von $\mathrm{k}=0,78$ zu einem sicher zu kleinen Maximalwert des Alters der Atmosphäre von 1,6.109 a schon nach dem klassischen Zerfallsgesetz führt.

Im dritten Abschnitt wurden Konsequenzen des Zerfallsgesetzes (2) für Altersbestimmungen nach der Rubidium-Strontium-Methode diskutiert. Hierbei wird festgestellt, daß dieses Zerfallsgesetz experimentell mit den chemischen Befunden von $\mathrm{Hah} \mathrm{n}$ und Mitarbeitern an einigen Mineralien verträglich ist, wenn eine durch Messungen von $M \ddot{u}$ h l $h$ of $f$ und dem einen von uns bestätigte Halbwertszeit von $R b^{87}$ von $T=0,9.10^{11}$ a und das Weltalter im Einklang mit dem Befund aus dem $\mathrm{Hubble}$ - Effekt zu $\mathrm{t}=2,4.10^{\circ}$ a angenommen wird. Das Alter des von $\mathrm{Hahn}$ und Mitarbeitern untersuchten Polluzits von Varuträsk ergibt sich dann höher, nämlich zu 680 statt 530 Millionen Jahren.

Im letzten Abschnitt wird auf die prinzipielle Möglichkeit hingewiesen, aus den Intensitätsverhältnissen sehr alter pleochroitischer und Verfärbungshöfe von Körpern der Thoriumreihe Schlüsse über die Konstanz des $\beta$-Zerfalls zu ziehen.
\end{abstract}

\section{Das Zerfallsgesetz für $\beta$-Strahlung und K-Einfang}

T n Zusammenhang mit den kürzlich angestellten Überlegungen ${ }^{2}$ über die Entstehung des Kosmos und die Zeitabhängigkeit gewisser Naturkonstanten wurde im Anschluß an Blackett ${ }^{3}$ auch eine zeitliche Abnahme der Zerfallskonstanten des Mesons, der positiven und negativen $\beta$-Strahler und des K-Einfangs - nicht aber der $\alpha$-Strahler - , proportional der reziproken Wurzel aus der vom Zeitpunkt der Weltentstehung gerechneten Zeit $\mathrm{t}^{-\mathbf{1} / \mathbf{2}}$ angenommen. Durch ein Versehen ist in der erwähnten Arbeit ${ }^{2}$ aber die Betrachtung für einen Gang von $\lambda$ proportional $t^{+1 / 2}$ gegeben, die dort $\mathrm{zu}$

1 Eine kurze Mitteilung über den Inhalt vorliegender Arbeit erscheint in den Göttinger Nachrichten. einem nicht mit den beabsichtigten Annahmen verträglichen Abklingungsgesetz der $\beta$-Strahler führt. Nimmt man entsprechend der ursprünglichen Annahme an, daß

$$
\frac{d \mathrm{~N}}{\mathrm{dt}}=-\mathrm{C} \cdot \mathrm{t}^{-1 / 2} \cdot \mathrm{N}
$$

ist, so folgt daraus, wenn im Moment der Weltentstehung eine Anzahl $\mathrm{N}_{0}$ von Atomen eines $\beta$-Strahlers vorhanden waren oder vorhanden gewesen wären, daß die zur Zeit $t$ noch davon vorhandene Anzahl gegeben ist durch das Zerfallsgesetz

$$
\mathrm{N}(\mathrm{t})=\mathrm{N}_{\mathrm{o}} \cdot \exp \left(-2 \mathrm{C} \cdot \mathrm{t}^{1 / 2}\right),
$$

2 P. Jordan, Physik. Z. 45, 183 [1944].

3 P. M. S. Blackett, Nature [London] 144, 30 [1939]. 
wobei die heute gemessene Zerfallskonstante $\lambda_{1}$ sich zu

$$
\lambda_{1}=\mathrm{C} \cdot \mathrm{t}^{-1 /}
$$

ergibt, und $t_{1}$ das heutige Alter der Welt ist.

Das mit der jetzt gemessenen Zerfallskonstante unter der Voraussetzung von deren Konstanz ermittelte „klassische“ Alter a* eines Minerals ist, wenn $\mathrm{N}$ die jetzt vorhandene Atomzahl des $\beta$ - (bzw. K-) aktiven Elements, $M$ die des stabilen Folgeprodukts ist, gegeben durch

$$
\mathrm{a}^{*}=\mathrm{C}^{-1} \mathrm{t}_{1}^{1 / 2} \ln (1+\mathrm{M} / \mathrm{N}) .
$$

Unter Verwendung des Zerfallsgesetzes (2) berechnet sich, wenn $t_{0}$ die vom Beginn der Welt bis zur Bildung des Minerals verflossene Zeit, also $\left(\mathrm{t}-\mathrm{t}_{0}\right)=\mathrm{a}$ das wahre Alter des Minerals ist, aus

$$
\begin{aligned}
& \mathrm{N}\left(\mathrm{t}_{1}\right)+\mathrm{M}\left(\mathrm{t}_{1}\right)=\mathrm{N}\left(\mathrm{t}_{\mathrm{o}}\right)=\mathrm{N}_{\mathrm{o}} \cdot \exp \left(-2 \mathrm{C} \cdot \mathrm{t}_{\mathrm{o}}^{1 / 2}\right) \\
& \mathrm{N}\left(\mathrm{t}_{1}\right)=\mathrm{N}_{\mathrm{o}} \cdot \exp \left(-2 \mathrm{C} \cdot \mathrm{t}_{1}^{1 / 2}\right)
\end{aligned}
$$

die Beziehung

$$
\ln (1+\mathrm{M} / \mathrm{N})=2 \mathrm{C} \cdot\left(\mathrm{t}_{1}^{1 / 2}-\mathrm{t}_{\mathrm{o}}^{1 / 2}\right)
$$

und damit ergibt sich für das klassische Alter a*:

$$
\mathrm{a}^{*}=2\left(\mathrm{t}_{1}-\sqrt{\left.\mathrm{t}_{\mathrm{o}} \mathrm{t}_{1}\right)}=2\left[\mathrm{t}_{1}-\sqrt{\mathrm{t}_{1}\left(\mathrm{t}_{1}-\mathrm{a}\right)}\right] .\right.
$$

Bezeichnen wir das Verhältnis des wahren bzw. des „klassischen“ Alters des Minerals zum heutigen Weltalter mit $\alpha$ bzw. $\beta$ :

$$
\alpha=\mathrm{a} / \mathrm{t}_{1} ; \quad \beta=\mathrm{a}^{*} / \mathrm{t}_{1},
$$

so wird das aus dem $\beta$-Zerfall ermittelte scheinbare relative Alter des Minerals $\beta$ unter Annahme des Zerfallsgesetzes (2) als Funktion des wahren, aus dem $\alpha$-Zerfall ermittelten relativen Alters des gleichen Minerals

$$
\beta=2(1-\sqrt{1-c}),
$$

und für Mineralien, deren wahres Alter klein gegen das Weltalter ist, wird

$$
\begin{gathered}
\frac{\beta}{a}=1+\frac{1}{4} a+\frac{1 \cdot 3}{4 \cdot 6} a^{2}+\frac{1 \cdot 3 \cdot 5}{4 \cdot 6 \cdot 8} a^{3}+ \\
+\frac{1 \cdot 3 \cdot 5 \cdot 7}{4 \cdot 6 \cdot 8 \cdot 10} a^{4}+. .
\end{gathered}
$$

Der Verlauf von $\beta$ gegen $\alpha$ gemäß (7) ist in Abb. 1 wiedergegeben, ebenso der Verlauf von $\beta / \alpha$ gegen $\alpha$; das scheinbare $\beta$-Alter $\beta$ muß dann im Grenzfall eines Minerals, das so alt ist, wie die Welt selbst, gerade doppelt so groß scheinen, wie das wahre $\alpha$-Alter; für relativ junge Mineralien wird aber die Entscheidung noch außerhalb der gegenwärtigen Genauigkeitsgrenzen liegen.

Wir wollen im folgenden einige Möglichkeiten der experimentellen Prüfung des Zerfallsgesetzes (2) diskutieren, um die Richtung anzugeben, in der die Annahme der zeitlichen Inkonstanz des $\beta$-Zerfalls

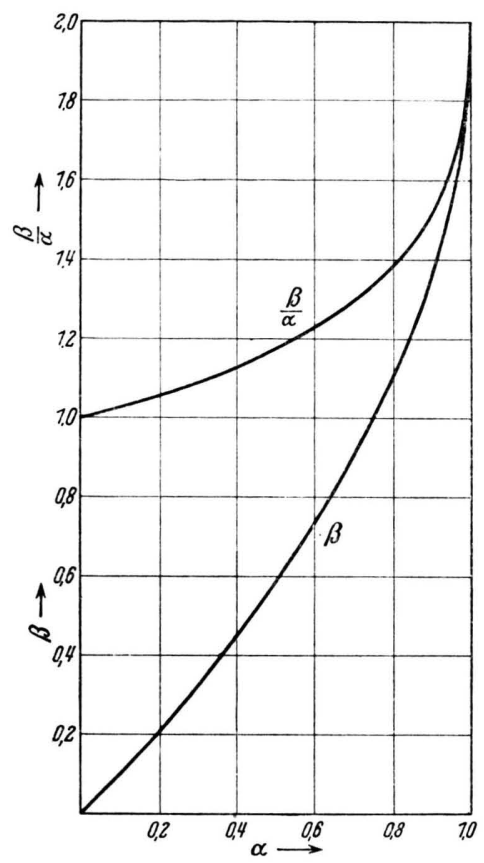

Abb. 1. Diskrepanz zwischen scheinbarem und wahrem Alter eines Minerals, nach Zerfallsgesetz (2) in Abhängigkeit vom relativen wahren Alter des Minerals $\alpha$.

$$
\begin{aligned}
& a=\frac{\text { wahres Alter }}{\text { Weltalter }} \\
& \beta=\frac{\text { scheinbares } \beta \text {-Strahlalter }}{\text { Weltalter }}
\end{aligned}
$$

Mineralien, deren Alter gerade gleich dem Weltalter ist, scheinen auf Grund des $\beta$-Zerfalls gerade doppelt so alt, wie sie wirklich sind.

und des K-Einfangs Diskrepanzen mit der bisherigen Auffassung erwarten läßt. Wir denken hierbei im wesentlichen an 3 Punkte.

1. Diskrepanz zwischen experimenteller Zerfallskonstante des K-Einfangs des Kaliumisotops $\mathrm{K}^{40}$ und der vorhandenen Menge des Argonisotops gleicher Masse;

2. Altersbestimmungen nach der Strontiummethode;

3. Änderung des Abzweigungsverhältnisses an den C-Produkten der radioaktiven Zerfallsreihen 
und des entsprechenden Intensitätsverhältnisses an pleochroitischen Höfen der Thoriumreihe.

\section{Die $\mathrm{K}-\mathrm{E}$ inf angung von $\mathrm{K}^{40}$}

Die große Häufigkeit des Argonisotops $\mathrm{A}^{40}$ kann nach v. We iz säcke ${ }^{4}$ durch K-Einfangung des Isotops $\mathrm{K}^{40}$ erklärt werden. v. Weizsäcker versucht das - übrigens auch nach (2) naturgemä $ß$ konstante - Abzweigungsverhältnis $z$ wischen $\beta$-Zerfall und K-Einfang abzuschätzen, indem er die Menge des in der Atmosphäre vorhan* denen Argonisotops $\mathrm{A}^{40}$ in Beziehung zu der noch in einer Schicht der Dicke d vorhandenen Menge an $\mathrm{K}^{40}$ setzt. Ist die heute gemessene resultierende Zerfallskonstante des $\mathrm{K}^{40}$

$$
\lambda=\lambda_{\mathrm{K}}+\lambda_{-},
$$

und das Verhältnis der auf dem Wege des K-Einfangs pro Zeiteinheit zerfallenden Atome zu den insgesamt zerfallenden Atomen

$$
\mathrm{k}=\lambda_{\mathrm{K}} / \lambda,
$$

so findet v. Weizsäcker unter der freilich recht willkürlichen Annahme, daß d $=16 \mathrm{~km}$, und die Hälfte des zu Beginn der Erdgeschichte vorhandenen $\mathrm{K}^{40}$ heute noch vorhanden sei, die Abschätzung $\mathrm{k}=1 / 3$. Für den Wert von $\lambda$ nimmt er dabei 3,46 $\cdot 10^{-10} \mathrm{a}^{-1}$ an. Um den Zusammenhang zwischen $k, \lambda$, dem ,Alter der Atmosphäre" $\left(t-t_{0}\right)$ und dem Weltalter $t$ anzugeben, wenden wir die Formeln des dualen Zerfalls an und finden zur Zeit t für das Verhältnis $\mathrm{x}$ von $\mathrm{A}^{40}$ zum noch vorhandenen $\mathrm{K}^{40}$ in Atomzahlen nach der klassischen Zerfallstheorie

$$
\mathrm{x}=\mathrm{k}\left[\mathrm{e}^{\lambda\left(\mathrm{t}-\mathrm{t}_{\mathrm{o}}\right)}-1\right],
$$

und entsprechend nach dem Zerfallsgesetz (2)

$$
\mathrm{x}=\mathrm{k}\left[\mathrm{e}^{2 \hat{\lambda}\left(\mathrm{t}-\sqrt{\mathrm{t}_{\mathrm{o}}}\right)}-1\right] .
$$

Wir wollen nun versuchen, die mit zum Teil bewußt willkürlichen Annahmen durchgeführte Abschätzung v. Weizsäckers auf Grund neuerer

${ }^{4}$ v. Weizs äcker, Physik. Z. 38, 623 [1937].

5 C. W. Correns, private Mitteilung. Praktisch zur gleichen Zahl kommt auf Grund etwas anderer Uberlegungen V.M. Goldschmidt, Geochem. Verteilungsges. IX, S. 11. Für zahlreiche anregende Diskussionen und Ratschläge über diesen Gegenstand möchten wir Hrn. C. W. C o r r en s unseren besten Dank aussprechen.
Daten $\mathrm{zu}$ verbessern, und machen zu diesem $\mathrm{Z}$ weck folgende Annahmen:

1. Praktisch die gesamte in der Atmosphäre vorhandene Menge an $\mathrm{A}^{40}$ ist durch $\mathrm{K}$-Einfang aus $\mathrm{K}^{40}$ entstanden.

2. Wir machen die Annahme, das $\mathrm{A}^{40}$ der Atmosphäre entstamme im wesentlichen demjenigen Teil der Erdrinde, der wenigstens einmal den Verwitterungsvorgang (Primärgestein, Weltmeer, Sedimentgestein) durchgemacht hat. Wir setzen also die Menge $\mathrm{A}^{40}$ mit derjenigen Menge $\mathrm{K}^{40}$ in $\mathrm{Be}-$ ziehung, die noch im Weltmeer und in den Sedimentgesteinen vorhanden ist. Unsere Annahme bedeutet, daß wir die von den primären Eruptivgesteinen abgegebene und in den Sedimentgesteinen (etwa in fein zermahlenem Glimmer und Feldspat) zurückgehaltenen Mengen an $\mathrm{A}^{40}$ gegenüber dem Gehalt der Atmosphäre vernachlässigen wollen. Die Menge an primären Eruptivgesteinen, die das Ausgangsmaterial für die im Ozean gelöste oder auf den Sedimentgesteinen niedergeschlagene Gesteinsmenge ist, läßt sich in einfacher Weise nach $\mathrm{C}$ orren $\mathrm{s}^{5}$ aus dem Gehalt an Kalium und Natrium in den Eruptivgesteinen, dem Ozean und den Sedimentgesteinen berechnen. Die so erhaltene Menge ist $156 \mathrm{~kg} / \mathrm{cm}^{2}$ Eruptivgestein als Ausgangsmaterial für den Gehalt an $\mathrm{A}^{40}$ in der Atmosphäre. Nimmt man mit $\mathrm{Clarke}$ und W a$\mathrm{sh}$ ing to ${ }^{6}$ als mittleren Gehalt an Kalium in den primären Eruptivgesteinen 2,6 Gew.\% an, so ergibt sich für Ozean und Sedimentgesteine zusammen ein Gehalt von $4,06 \mathrm{~kg} / \mathrm{cm}^{2}$ an Kalium und $0,49 \mathrm{~g} / \mathrm{cm}^{2}$ an $\mathrm{K}^{40}$. Dem stehen in der Atmosphäre $12,9 \mathrm{~g} / \mathrm{cm}^{2}$ an $\mathrm{A}^{40}$ gegenüber. Unter den genannten Annahmen wird also

$$
\mathrm{x}=26, \tilde{\mathrm{o}},
$$

d. h. die ursprünglich vorhandene Menge an $\mathrm{K}^{40}$ ist also schon zum größten Teil abgeklungen. Da nach (9) und (10) sich

$$
(1-\mathrm{k}) \lambda=\lambda
$$

ergibt, so läßt sich, wenn wir den Wert von B ra mle y und $\mathrm{B}$ rew e ${ }^{7} \mathrm{~T}=14,2.10^{8} \mathrm{a}$ als richtig ansehen, sowohl nach (11) wie nach (12) der Wert von $\mathrm{k}$ berechnen, wenn man für das Alter der Erde $\left(t-t_{0}\right)$ einen Wert innerhalb der heute als richtig anzusehenden Grenze

$$
2 \cdot 10^{9} \mathrm{a}<\mathrm{t}-\mathrm{t}_{\mathrm{o}}<6 \cdot 10^{9} \mathrm{a}
$$

${ }^{6}$ Clarke, Data on Geochemistry 1925.

7 B r a mley u. B rew er, Physic. Rev. 53, 502 [1938]. 
annimmt. Während im Fall des klassischen Zerfallsgesetzes das Alter der Welt bei Entstehung der Erde (oder richtiger bei der Absonderung der Atmosphäre) nicht in die Rechnung eingeht, setzt die Berechnung nach dem Zerfallsgesetz (2) eine Annahme über $\mathrm{t}_{0}$ selbst voraus. Wir wollen nun hier den Zusammenhang zwischen $\mathrm{k}$ und dem Alter der Atmosphäre für den Extremfall berechnen, daß wir das Alter der Erde dem der Welt ungefähr gleichsetzen können, also $t_{0}=0$ annehmen. Die Voraussage nach (2) macht dann gegenüber der klassischen, aus dem $\beta$-Zerfall berechneten gerade einen

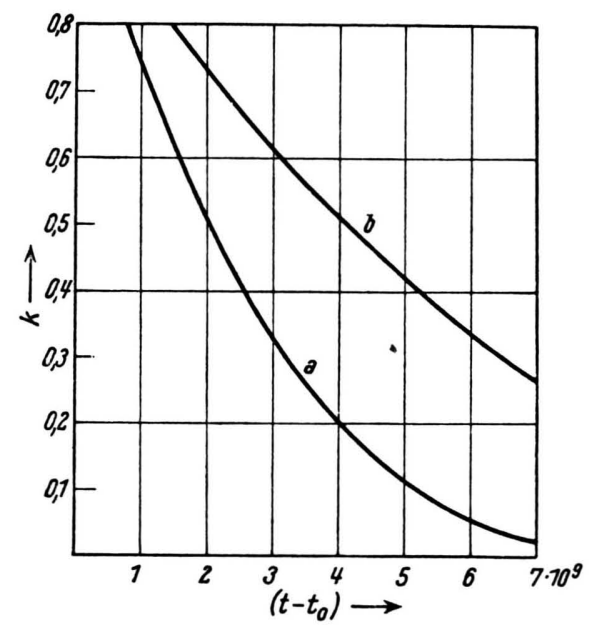

Abb. 2. Abzweigungsverhältnis $\mathrm{k}$ von $\mathrm{K}$-Einfang $\mathrm{zu}$ Gesamtzerfall des $\mathrm{K}^{40}$, berechnet unter der Voraussetzung, das $\mathrm{A}^{40}$ der Atmosphäre entstamme dem verwitterten Teil der Eruptivgesteine $\left(\mathrm{A}^{\mathbf{4 0}} / \mathrm{K}^{\mathbf{4 0}}=26,5\right)$ in Abhängigkeit vom angenommenen Alter der Atmosphäre, berechnet a) nach Formel (2), wenn $t_{0}=0$ angenommen wird, b) für konstanten Zerfall.

Faktor ${ }^{1 / 2}$ im Alter aus. Abb. 2 gibt k in Abhängigkeit vom angenommenen Alter der Atmosphäre $\left(t-t_{0}\right)$ auf Grund der beiden Zerfallsgesetze an. Für einen plausiblen Mittelwert, etwa $\left(t-t_{0}\right)$ $=3,5 \cdot 10^{9} \mathrm{a}$, folgt also klassisch $\mathrm{k}=0,56$ und für $\mathrm{t}_{0}=0$ nach (2) $\mathrm{k}=0,26$, während für jeden höheren Wert von $t_{0}$ die Aussagen nach (2) sich den klassischen nähern müssen. In der Tat geht ja die Kurve für $k$ in Abhängigkeit von $\left(t-t_{0}\right)$ aus der klassischen durch eine Maßstabsverzerrung um den Faktor $\beta / \alpha$ hervor, der durch Abb. 1 gegeben ist. Schließlich wurde kürzlich von Thompson und $\mathrm{Row}$ o ands ${ }^{8}$ eine freilich recht ungenaue experimentelle Abschätzung von $\mathrm{k}$ durch eine tat-

$s$ F. C. Thompson u. S. Rowlands, Nature [London] 152, 103 [1943]. sächliche Beobachtung der mit der K-Einfangung von $\mathrm{K}^{40}$ verbundenen K-Strahlung mitgeteilt. Diese Verfasser finden das Verhältnis der Häufigkeit von $\mathrm{K}$-Einfangung zu $\beta$-Emission etwa gleich $3,5: 1$, also $\mathrm{k}=0,78$. Setzt man diesen Wert in die beiden Gleichungen (11) und (12) ein, und nimmt wieder $\mathrm{x}=26,5$ und $\mathrm{T}=1,42 \cdot 10^{9} \mathrm{a}$ an, so erhält man einen offensichtlich zu kleinen Wert für das Alter der Atmosphäre, nämlich bei Konstanz des $\beta$-Zerfalls $\left(\mathrm{t}-\mathrm{t}_{0}\right)=1,6.10^{9} \mathrm{a}$ und bei abnehmendem $\beta$-Zerfall für $t_{0}=0$ die Hälfte, also $0,8 \cdot 10^{9} \mathrm{a}$.

Man wird hieraus schließen müssen, daß entweder der experimentelle Wert von $\mathrm{k}$ nach T h o m pson und Rowlands oder $\mathrm{x}$ zu hoch ist, oder die oben gemachte Voraussetzung, das $\mathrm{A}^{40}$ der Atmosphäre entstamme im wesentlichen dem $\mathrm{K}^{40}$ aus den in Ozean und den Sedimentgesteinen verarbeiteten Teilen der Erdrinde, unrichtig ist. Es soll ausdrücklich darauf hingewiesen werden, daß eine so hohe Wahrscheinlichkeit der K-Einfangung, wie die genannten Autoren angeben, durchaus nachweisbar sein müßte. Ein etwa dem Kambrium entstammender Feldspat von 14 Gew.\% Kaliumgehalt müßte bei einem Alter von 500 Millionen Jahren, wenn er nicht sein Argon verloren hat, schon nach der klassischen Zerfallstheorie 1,51.10-2 $\mathrm{cm}^{3}$ $\mathrm{A}^{40} / \mathrm{g}$, nach (2) um 15-70\% mehr enthalten, je nachdem, wie man das Alter der Welt zwischen $6.10^{9}$ und $2.10^{9}$ Jahren ansetzt.

\section{Die Rubidiummethode}

Wesentlich günstiger liegen die Aussichten für eine Prüfung der Konstanz des $\beta$-Zerfalls bei Anwendung der Rubidiummethode von $\mathrm{Hahn}$, Stra $ß \mathrm{mann}$, M a t t a u ch und Ewald ${ }^{\boldsymbol{9}}$. Die Ursache hierfür liegt darin, daß als Zerfallsprodukt des Rubidiumzerfalls ein im gewöhnlichen Strontium seltenes Strontiumisotop entsteht, so daß eine massenspektroskopische Analyse auch bei Anwesenheit von geringen Beimengungen von accidentellem Strontium ohne Schwierigkeiten die Messung des Verhältnisses $\mathrm{Sr}^{87} / \mathrm{Rb}^{87}$ gestattet, während im analogen Fall des $\mathrm{K}^{40} / \mathrm{Ca}^{40}$ die Verhältnisse viel ungünstiger liegen. Im Falle des von $\mathrm{Hah}$ u und Stra ßmann untersuchten Lepidoliths vom Großen Bärensee, dessen geologisches Alter aus $\alpha$-Zerfallsdaten der gleichen Schicht von L a ne zu 1,975 .10 ${ }^{9} \mathrm{Jahren}$ geschätzt wurde, hat Mat-

9 O. Hahn, F. Straßmann, J. Mattauch, H. Ew ald, Forsch. u. Fortschr. 18, Nr. 35/36, S. 1 [1942]. 
tauch sogar zeigen können, daß das darin vorhandene Strontium aus reinem $\mathrm{Sr}^{87}$ besteht, also keine nachweisbare Beimengung von accidentellem Strontium vorliegt. Die genannten Verfasser haben nun die Halbwertszeit von $\mathrm{Rb}^{87}$ umgekehrt aus dem hier gefundenen Verhältnis $\mathrm{Rb}^{87} / \mathrm{Sr}^{87}$ und dem als bekannt vorausgesetzten Alter unter Annahme der Konstanz des $\beta$-Zerfalls berechnet und für das $R b^{8 \tau}$ den Wert $\mathrm{T}=6,3 \cdot 10^{10}$ a gefunden. Dieser Wert stimmt gut mit einer Messung von $\mathrm{Hahn}$ und $\mathrm{Ro}$ the $\mathrm{n} \mathrm{bach}{ }^{10}$ uiberein, die nach Umrechnung auf $\mathrm{Rb}^{87} \mathrm{~T}=5,2.10^{10}$ a ergeben hatte. Aus diesem Befund wird man zunächst schliefen müssen, daß, wenn man die Fehlergrenzen der angeführten Messungen in Betracht zieht, entweder der $\beta$-Zerfall überhaupt konstant ist, oder, wenn man das Zerfallsgesetz (2) als richtig ansieht, das Weltalter groß gegen das Alter des Minerals, also $t \geqslant 6.10^{10} \mathrm{a}$ angenommen werden muß, sicher also größer als der maximale Wert von $t=2.10^{9}$ bis $3.10^{9} \mathrm{a}$, den die Astrophysiker auf Grund des $\mathrm{Hubble-Effekts}$ zulassen.

Doch lassen die experimentellen Daten von $\mathrm{H}$ a h n und Stra $ß \mathrm{~m}$ a n n ebensogut noch eine andere Deutung zu, wenn man sie mit einer mit dem Zählrohr von $\mathrm{W}$. M $\ddot{u} \mathrm{hlh}$ of $\mathrm{f}^{11}$ durchgeführten Messung der Halbwertszeit des Rubidiums vergleicht. Dieser fand - umgerechnet auf $\mathrm{Rb}^{87}$ - den Wert $\mathrm{T}=1,2 \cdot 10^{11} \mathrm{a}$, also ungefähr den doppelten Wert. Hahn und Straßmann lehnen diesen Wert ab, weil er - unter der Voraussetzung des konstanten $\beta$-Zerfalls - zu dem sicher zu hohen Alter von etwa $4.10^{9}$ Jahren für den gemessenen Lepidolith führen würde. Vom Standpunkt der durch (2) gekennzeichneten Inkonstanz des $\beta$-Zerfalls ist das aber gerade die Richtung, in der eine Abweichung zu erwarten wäre.

Wenn experimentelle Einwände gegen die $\mathrm{M}$ ü h l$\mathrm{h}$ off sche Messung vorliegen, so wären diese zweifellos darin $\mathrm{zu}$ suchen, daß dieser sich auf Uran als Vergleichssubstanz bezieht, und man nicht ganz sicher sein kann, ob, wenigstens für Teilchen, die unter sehr schrägem Winkel emittiert werden, die von ihm angewandte Schichtdicke der Uranpräparate von einigen Zehntel $\mathrm{mg} / \mathrm{cm}^{2}$ als hinreichend dünn auch für $\alpha$-Strahlen betrachtet werden kann, während er ohne Bedenken die Hälfte der von ihm gemessenen Impulszahlen als durch $\alpha$-Strahlen ver-

10 O. Hahn u. Rothenbach, Physik. Z. 20, 194 [1919].

11 W. M ühlhoff, Ann. Physik (5) 7, 205 [1930]. ursacht deutet und die übrigen unter Voraussetzung gleichen Zählrohrnutzeffekts für die 3-Strahlen des $\mathrm{UX}_{1}$ und $\mathrm{UX}_{2}$ zu je $50 \%$ einem dieser beiden $\beta$-Strahler zuordnet.

Eigene, von einem von uns unternommene Versuche, die infolge der Zeitereignisse leider nicht abgeschlossen werden konnten, ließen für das Verhältnis der pro see und g emittierten Zahl der $\beta$-Teilchen in unendlich dünner Schicht etwa den Wert $\mathrm{Rb}: \mathrm{K}=18 \pm 2$ vermuten, während $\mathrm{M} \ddot{\mathrm{u}} \mathrm{h} \mathrm{l}$ h off hierfür $350 / 23=15,2$ findet. Benützt man diesen letzten Mühlhoffschen Wert und bezieht seine Messung nicht auf Uran, sondern auf Kalium, so erhält man als Halbwertszeit des $\mathrm{Rb}^{87}$ nach unseren Messungen 8,0 $\pm 0,5 \cdot 10^{10}$ a, nach $\mathrm{M} u ̈ h l-$ h off selbst 9,2 $10^{10}$ a, wenn man zur Eichung die modernste vorliegende Messung der Halbwertszeit von Kalium, die von $\mathrm{Bram} \mathrm{ley} \mathrm{und} \mathrm{Brew}$ e ${ }^{7}$ mit $1,42.10^{9}$ a für $\mathrm{K}^{40}$, benutzt. Beide Werte liegen zwischen dem von $\mathrm{M} \ddot{\mathrm{u}} \mathrm{hlh}$ off und den von $\mathrm{Hahn}$ und Rothenbach gemessenen Werten, und wir wollen hier mit einem Mittelwert von $\mathrm{T}=9.10^{10} \mathrm{a}$ für $\mathrm{Rb}^{87}$ rechnen. Damit erhalten wir aber für das scheinbare Alter des von $\mathrm{Hahn}$ und Mitarbeitern gemessenen Lepidoliths den Wert $\mathrm{a}=2,86.10^{9}$ Jahren. Entschließt man sich, diesen Unterschied als reell anzusehen, so erhalten wir vom Standpunkt des in (2) formulierten Zerfallsgesetzes die in (6) definierte Größe $\beta / \alpha=1,43$ und können versuchsweise hieraus auf Grund von (7) an der in Abb. 1 gegebenen Kurve $\alpha=0,84$ und damit das heutige Alter der Welt zu $t=2,4.10^{9}$ a abschätzen. Dieser Wert liegt zwar etwas niedriger als die heute meist angenommenen Werte, wäre aber doch gerade noch eben mit den aus dem $\mathrm{Hu}$ bble-Effekt gewonnenen Daten vereinbar. Freilich steht unsere Abschätzung, wie ja auch alle aus dem $\mathrm{Hubble-}$ Effekt gewonnenen Werte, mit dem von $\mathrm{Paneth}$ und Mitarbeitern ${ }^{12}$ gefundenen Alter eines Meteoriten von $t=7,2 \cdot 10^{9} \mathrm{a}$ in Widerspruch.

Nimmt man, wie wir es getan haben, $T=9,0.10^{1 \mathrm{j}} \mathrm{a}$ als Halbwertszeit für $\mathrm{Rb}^{87}$ an, so ändern sich freilich auch alle andern nach der Rb-Methode gewonnenen Altersbestimmungen, und diese Diskrepanzen werden um so stärker, um je jüngere Mineralien es sich handelt, weil bei diesen die Diskrepanz zwischen $\alpha$ und $\beta$ geringer wird. Für den bekannten schwedischen Polluzit von Varuträsk, für den

12 W. J. Arrol, R. B. Jacobi u. F. A. Paneth, Nature [London] 149, 235 [1930]. 
$\mathrm{Hahn}$ und Straßmann ein Alter von $5,30.10^{8}$ a angeben, erhalten wir auf Grund der hier gemachten Voraussetzungen ein scheinbares Alter von $7,58 \cdot 10^{8} \mathrm{a}$, woraus mit dem angenommenen Wert des Weltalters $t=2,4 \cdot 10^{9}$ a für den Polluzit ein wahres Alter von 680 Millionen Jahren folgt, was mit seiner geologischen Herkunft noch verträglich sein dürfte.

\section{Dualer Zerfall und Verfärbungshöfe}

Zuletzt möge noch auf eine weitere prinzipielle Möglichkeit hingewiesen werden, die Konstanz des $\beta$-Zerfalls zu prüfen, bei der es sich um einen Vergleich von Wirkungen von $\alpha$-Strahlen handelt. Bekanntlich treten ja in den natürlichen radioaktiven Reihen, und zwar beim Ac und bei den C-Produkten der drei Reihen, Fälle von dualem, von gleichzeitigem $\alpha$ - und $\beta$-Zerfall auf. Gilt nun das Zerfallsgesetz (2), so ist das Verzweigungsverhältnis ebenfalls als zeitabhängig zu betrachten, wobei die Zerfallskonstante des $\alpha$-Zerfalls als konstant anzusehen ist. Das Abzweigungsverhältnis

$$
\mathrm{x}=\frac{\lambda_{\beta}}{\lambda_{a}+\lambda_{\beta}}
$$

einer dual zerfallenden Substanz zur Zeit $t$ nach Weltbeginn ist daher, wenn $\mathrm{x}_{1}$ sein heutiger Wert ist, gegeben durch

$$
x(t)=\frac{x_{1}}{x_{1}+\left(1-x_{1}\right) \sqrt{t / t_{1}}} .
$$

Danach müßten z. B. von den Elementen Ac, AcC, $\mathrm{RaC}$ und Th C, die heute zu 99 bzw. 0,32, 99,96 und $65 \%$ auf dem $\beta$-Wege zerfallen, die entsprechenden Werte für $\mathrm{x}$, wenn wir wieder $\mathrm{t}_{1}=2,4.10^{9} \mathrm{a}$ annehmen, vor $10^{9}$ Jahren 99,3 bzw. 0,42, 99,97 und 71\% betragen haben, und entsprechend muß auch die Zahl der im radioaktiven Gleichgewicht von den Produkten des dualen $\beta$-Zerfalls, dem Ac K bzw. den $C^{\prime}$-Körpern pro sec emittierten $\alpha$-Teilchen herabgesetzt gewesen sein. Glücklicherweise sind nun die Reichweiten der von den $C^{\prime}$-Körpern emittierten
$\alpha$-Strahlen größer als die aller anderen $\alpha$-Strahlen der entsprechenden Reihen. Wir besitzen nun in den bekannten radioaktiven pleochroitischen und Verfärbungshöfen einiger Mineralien tatsächlich eine Art „photographischer" Aufnahmen des $\alpha$-Zerfalls über geologische Zeiträume hinweg. Wir müssen daher erwarten, daß das Schwärzungsverhältnis der, den verschiedenen $\alpha$-Strahlern einer Reihe entsprechenden Ringe dem Intensitätsverhältnis der betreflenden $\alpha$-Strahler entspricht, wie sie im radioaktiven Gleichgewicht vorhanden sind. Dabei geht für das Intensitätsverhältnis der C'-Körper zu den andern, z. B. den A-Körpern, der unter Berücksichtigung des zeitlichen Schwärzungsgesetzes gebildete zeitliche Mittelwert über $\mathrm{x}$ während des gesamten Alters des Minerals ein, nicht der heutige $\mathrm{x}$-Wert, so daß wir einen Unterschied im Schwärzungsverhältnis der äußersten Ringe zu den übrigen Ringen erwarten müssen, je nachdem es sich um ein sehr altes oder ein sehr junges Mineral handelt. Insbesondere muß sich auch das dem heutigen $\mathrm{x}$-Wert entsprechende Schwärzungsverhältnis der Ringe durch künstliche Impfung des Minerals mit einem entsprechend starken radioaktiven Kern herstellen lassen, wir dies von Rutherford und $J_{\text {oly }}{ }^{13}$ zum Zweck der Zeiteichung von Verfärbungshöfen durchgeführt worden ist. Praktisch kämen hierbei freilich nur solche Höfe in Frage, die von radioaktiven Höfen der Thoriumreihe herrühren, denn nur in diesem Falle ist das Abzweigungsverhältnis x nach (16) hinreichend empfindlich gegen die zeitliche Änderung des $\beta$-Zerfalls und gleichzeitig groß genug, um einer Messung zugänglich zu sein. Während also bei einem heute hergestellten Hofsystem der Thoriumreihe das Schwärzungsverhältnis $65 \%$ beträgt, wird es im gleichen Mineral unter Annahme eines Schwar z $\mathrm{schild-Exponenten} \gamma=1$ bei einem Alter von 1000 bzw. 500 Millionen Jahren $67,9 \%$ bzw. $66,3 \%$ betragen, wenn wir wieder wie oben das Alter der Welt zu 2,4 .109 Jahren annehmen.

13 J. Joly u. E. Rutherford, Philos. Mag. J. Sci. 25, $644[1913]$. 